\title{
Model-based segmentation and recognition of anatomical brain structures in 3D MR Images
}

\author{
Olivier Cuisenaire ${ }^{1}$, Matthieu Ferrant ${ }^{1}$, Jean-Philippe Thiran ${ }^{2}$ and Benoît Macq ${ }^{1}$ \\ ${ }^{1}$ Université catholique de Louvain. (BE) \\ ${ }^{2}$ Ecole Politechnique Fédérale de Lausanne $(\mathrm{CH})$
}

Cuisenaire@tele.ucl.ac.be

\begin{abstract}
:
We propose a coarse to fine framework for the segmentation and recognition of structures in brain MR Images. We first coarsely segment the outer surface of the brain, which is used as the main criteria for the non-rigid registration of the MR image with a Computerized Brain Atlas. Then, we use the structures in the atlas as the initialization for active surfaces models. Those surfaces are deformed in order to minimize an energy depending both on the smoothness of the structure and on the image itself.
\end{abstract}

\section{Introduction:}

The recognition of structures in brain images is a major part of the processing work for the neuro-radiologist in numerous clinical applications. To help him, various atlases of the brain have been developed. Statistical atlases are based on the collection of data from hundredth of subjects and provide, for each voxel of the image, a probability that it belongs to one structure/tissue or another. On the other hand, deterministic atlases are based on a single subject, and provide a clear boundary for each structure, but a perfect mapping of such an atlas with an image is impossible given the high inter-subject variability of brains.

The accurate segmentation of brain structures is a complex task. Manual procedures for slice per slice 3D segmentation are highly time consuming while automatic procedures relying on local criteria can hardly do more than separate the main tissue types (white matter, gray matter, CSF, lesions,...). Moreover, the boundaries between anatomical structures can be conventional and do not always correspond to features of the MR Image.

We propose a framework combining both recognition and segmentation of these structures. We first use a global deformation to register a global model (deterministic atlas) of the brain to our image. The structures of interest from this model are then locally adapted to the image by using them as starting point for an active surface segmentation.

\section{Atlas registration:}

In [2], we propose an automated procedure to find the best parameters for the $3 \mathrm{D}$ second degree global transformation proposed and routinely used by Thurjfell et al. in [1] for registering the Computerized Brain Atlas (CBA) with an MR image. First, the outer surface of the brain is coarsely segmented from the MR image using mathematical morphology. Similarly to [3], we define our registration criteria as the distance between this surface $S_{\text {mob }}$ and the equivalent objet from our reference CBA model $S_{\text {ref }}$ as

$$
d\left(S_{r e f}, S_{m o b}\right)=\sum_{p_{1} \in S_{\text {mob }}} d\left(p_{1}, S_{r e f}\right) / \# S_{r e f}=\sum_{p_{1} \in S_{\text {mob }}} \min _{p_{i} \in S_{\text {ref }}} d\left(p_{1}, p_{2}\right) / \# S_{r e f}
$$

This is efficiently computed by pre-computing the distance $\mathrm{d}\left(\mathrm{p}, \mathrm{S}_{\mathrm{ref}}\right)$ from any pixel to the surface $\mathrm{S}_{\text {ref }}$ using the Distance Transform algorithms described in [5], a efficient implementation of the Euclidean Distance Transform proposed in [4]. The global transformation is then considered as the linear combination of 30 de-correlated base functions and the 30 coefficients of this transformation are optimized by a gradient-based minimization of the distance criteria. 


\section{Active Surface Model:}

In [6], Kass et al. define active contours models as parametric curbs $v(s)=(x(s), y(s))^{\mathrm{T}}$ where $s \in[0,1]$. These contours evolve to minimize an energy typically defined as

$$
E(v)=\int_{0}^{1} w_{1}(s)\left|\frac{\partial v}{\partial s}\right|^{2}+w_{2}(s)\left|\frac{\partial^{2} v}{\partial s^{2}}\right|+P(v(s)) d s
$$

a combination of smoothness terms w1 and w2 and of a third term depending on the image, typically such as $P(v)=-\nabla\left[G_{\sigma} * I(x, y)\right] \mid$, a decreasing function of the gradient of the smoothed image. The minimization of this energy is done locally and thus requires a good initialization of the contour to segment.

This model can be extended to 3D and active surfaces with two parameters ( $\mathrm{r}, \mathrm{s})$ and a similar energy to minimize. As proposed by Cohen in [7], the minimization can then be solved using finite element methods, provided we have a meshed description of our surface. The initial triangular meshes are provided by properly resampled structures from the CBA, to which the global transformation found at the previous section is applied. Such a structure is illustrated at figure 3 .

The use of CBA structures to initialize our surfaces actually provide us with more a priori information, which we can use to enrich the energetic model of our 3D snake: We can take into account the direction the gradient of the image should have on the surface boundaries. We can define relations between nearby objects to segment, ...

Finally, the local adaptation of the smoothness coefficient allow us to take chose the relative confidence we have in our model and in the information found in the MR image.

\section{Perspectives and Conclusion:}

The procedure we described can be summarized with the following schematic.

The framework described here a 3D non-linear correspondent to the concept of Kalman filtering in signal processing. We first have a strong confidence in our model and then progressively take into account the information coming from the data itself.

The major effort in our research is now on developing the active surface model so it can include the various a priori we have while using the structures from our brain atlas. Later we will obviously have to consider more closely the problem of the appropriate selection of coefficients for each term of this model, including their spatial and temporal variation according to the respective confidence
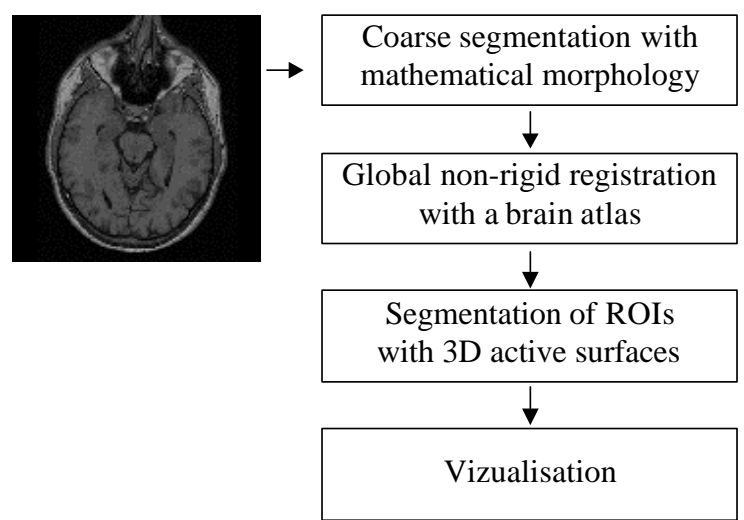
we have in our model and image.

\section{References:}

1. L. Thurjfell, C. Bohm, T. Greitz and L. Eriksson. Transformations and algorithms in a Computerized Brain Atlas. IEEE Transactions on Nuclear Sciences. vol. 40. pp. 1187-1191, 1993.

2. O. Cuisenaire, J.P. Thiran, B. Macq, C. Michel, A. De Volder and F. Marques. Automatic registration of 3D MR images with a computerized brain atlas, SPIE Medical Imaging 96, Newport Beach, SPIE vol. 1719, pp. 438-449

3. J.F Mangin, V. Froin, I. Bloch, B. Bendriem and J. Lopez-Krahe. Fast non-supervised 3D registration of PET and MR Images from the brain. Journal of Cerebral Blood Flow and Mechanism, vol. 14, pp. 749-792, 1994

4. P.E.Danielsson. Euclidean Distance Mapping, Computer Graphics and Image Processing 14, 1980, $227-$ 248.

5. O. Cuisenaire. Region Growing Euclidean Distance Transforms. ICIAP'97, Florence, September 97, Lecture Notes in Computer Science. 
6. M. Kass, A. Witkin and D. Terzopoulos. Snakes, active contour models. International Journal of Computer Vision, vol. 1, pp. 321-331, 1988

7. L.D. Cohen and I. Cohen. Finite element methods for active contour models and balloons for 2D and 3D images. IEEE Pattern Analysis and Machine Intelligence, 15, November 1993

\section{Illustrations:}

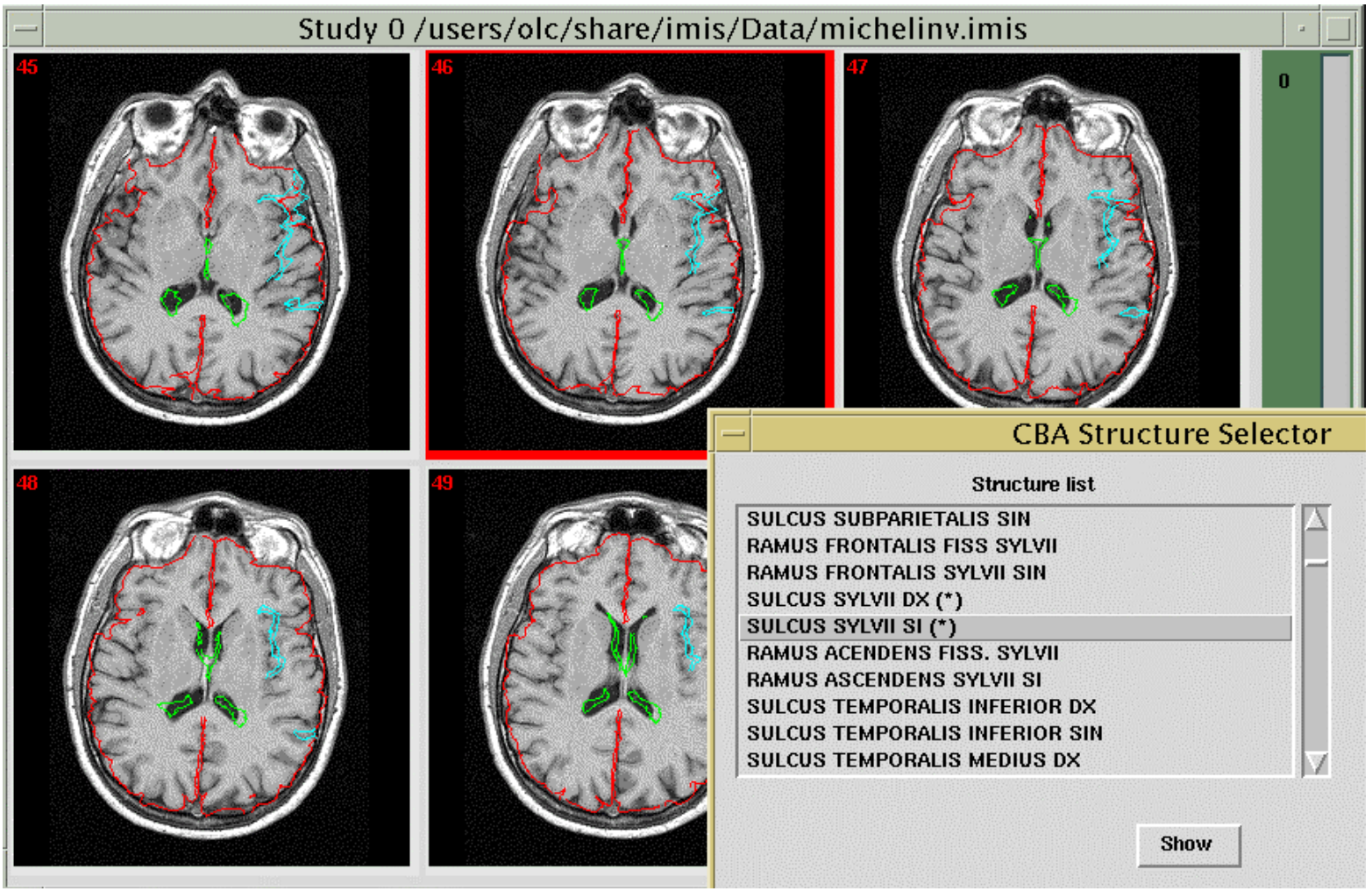

Fig. 1: Some structures of the Computerized Brain Atlas displayed after registration with a MR Image

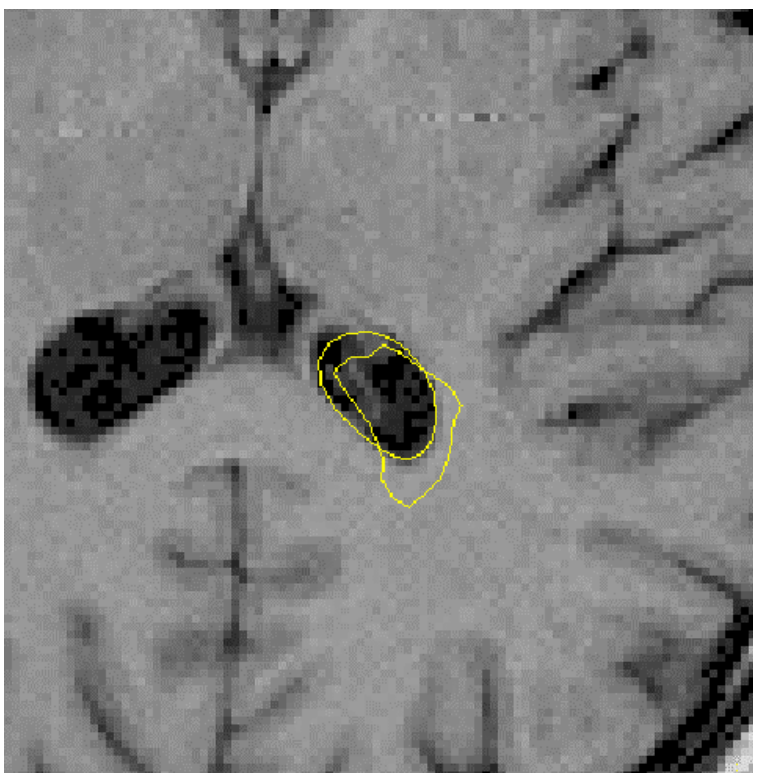

Fig 2: Initialization and result of the active contour (2D test model) segmentation of a part of the ventricular system

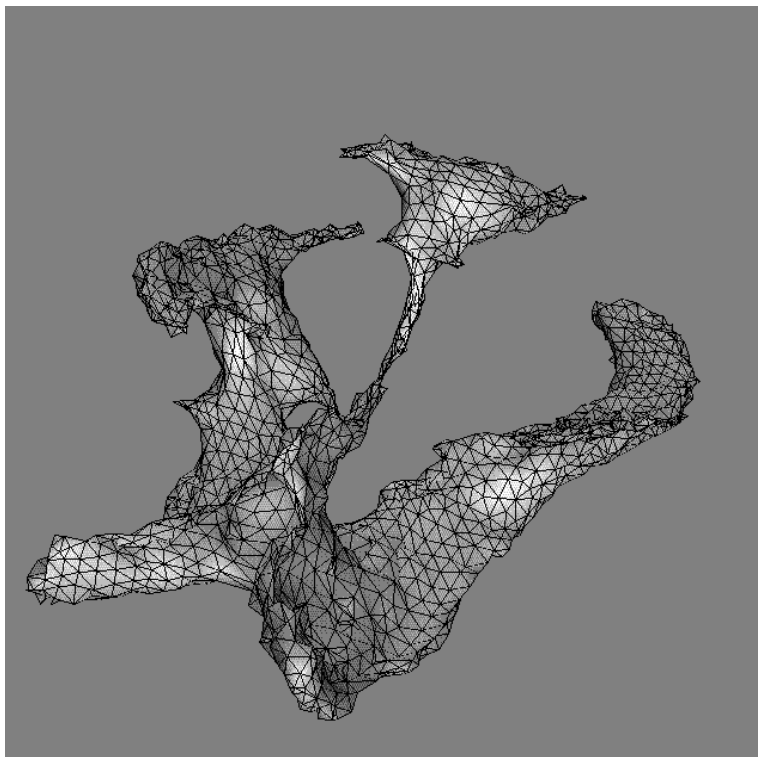

Fig 3: Triangular mesh for the ventricular system active surface. 